\title{
Human Auditory Brainstem Response to Temporal Gaps in Noise
}

\author{
Lynne A. Werner \\ Richard C. Folsom \\ Lisa R. Mancl \\ Connie L. Syapin \\ University of W ashington \\ Seattle
}

$\mathrm{G}$ ap detection is a commonly used measure of temporal resolution, although the mechanisms underlying gap detection are not well understood. To the extent that gap detection depends on processes within, or peripheral to, the auditory brainstem, one would predict that a measure of gap threshold based on the auditory brainstem response (ABR) would be similar to the psychophysical gap detection threshold. Three experiments were performed to examine the relationship between ABR gap threshold and gap detection. Thresholds for gaps in a broadband noise were measured in young adults with normal hearing, using both psychophysical techniques and electrophysiological techniques that use the ABR. The mean gap thresholds obtained with the two methods were very similar, although ABR gap thresholds tended to be lower than psychophysical gap thresholds. There was a modest correlation between psychophysical and ABR gap thresholds across participants.

ABR and psychophysical thresholds for noise masked by temporally continuous, high-pass, or spectrally notched noise were measured in adults with normal hearing. Restricting the frequency range with masking led to poorer gap thresholds on both measures. High-pass maskers affected the ABR and psychophysical gap thresholds similarly. Notched-noise-masked ABR and psychophysical gap thresholds were very similar except that low-frequency, notched-noise-masked ABR gap threshold was much poorer at low levels. The ABR gap threshold was more sensitive to changes in signal-to-masker ratio than was the psychophysical gap detection threshold. ABR and psychophysical thresholds for gaps in broadband noise were measured in listeners with sensorineural hearing loss and in infants. O $n$ average, both ABR gap thresholds and psychophysical gap detection thresholds of listeners with hearing loss were worse than those of listeners with normal hearing, although individual differences were observed. Psychophysical gap detection thresholds of 3-and 6-month-old infants were an order of magnitude worse than those of adults with normal hearing, as previously reported; however, ABR gap thresholds of 3-month-old infants were no different from those of adults with normal hearing. These results suggest that ABR gap thresholds and psychophysical gap detection depend on at least some of the same mechanisms within the auditory system.

KEY W ORDS: hearing development, temporal resolution, electrophysiologic measures

$\mathrm{T}$

emporal resolution refers to the auditory system's ability to follow rapid changes in the envelope of sound. As Viemeister and Plack (1993) point out, for speech and other acoustic communication sounds, "the temporal pattern of spectral changes is, essentially, the informational substrate" (p. 116). Thus, it is not surprising that the auditory system has evolved fine temporal resolution, nor is it surprising 
that deficits in temporal resolution are associated with deficits in speech understanding (e.g., Dreschler \& Leeuw, 1990; Dreschler \& Plomp, 1980; Phillips, 1999).

Temporal resolution is measured in various ways, including detection threshold for amplitude modulation (e.g., Viemeister, 1979), forward and backward masking (M oore, Glasberg, Plack, \& Biswas, 1988), and temporal order discrimination (Green, 1973). Gap detection is probably the most commonly used measure of temporal resolution. The gap detection threshold is the duration of the just-detectable interruption in a sound. In a sense, the gap detection threshold is not a "pure" measure of temporal resolution, as it also depends on intensity resolution. Gap detection is likely as popular a method as it is because it provides a description of temporal resolution based on a single threshold, whereas other methods require multiple threshold estimates. Another advantage is that gap detection is easy to measure in naive listeners, including infants. Gap detection threshol ds obtained from naive adults are cl ose to those obtained from well-trained listeners (e.g., Werner, Marean, Hal pin, Spetner, \& Gillenwater, 1992). Forrest and Green (1987) have reported that gap detection and the temporal modulation transfer function (based on amplitude modulation detection) yield similar estimates of temporal acuity.

Simple models of the auditory periphery-consisting, for example, of a bandpass filter followed by a halfwave rectifier and low-pass filter - have done a reasonable job of accounting for gap detection performance (although the required initial bandpass filter is extremely broad; Eddins, 1999; Moore, 1993). Several investigators have recorded the responses of single auditory neurons to sounds containing gaps and quantified the neural responses by various means to estimate "neural gap thresholds." ${ }^{1}$ The results of such an analysis will, of course, depend on the way in which the response is quantified as well as the criterion used to definethreshold. Nonetheless, such neural gap thresholds of auditory nerve fibers have been reported to be very similar to psychophysical gap detection thresholds in various species (e.g., Feng, Lin, \& Sun, 1994; Klump \& Gleich, 1991; Zhang, Salvi, \& Saunders, 1990). Gap thresholds of at least some single units in the central nervous system are also reported to be as low as gap detection threshol ds (Buchfellner, Leppel sack, Klump, \& Häusler, 1989; Eggermont, 1995, 1999; Walton, Frisina, Ison, \& O'Neill, 1997). Such findings have been taken to mean that gap detection is limited primarily by peripheral mechanisms, as reflected in the auditory nerveresponse.

It is also clear, however, that central processing is

\footnotetext{
"Throughout the paper we will use the term "gap threshold" to refer to thresholds for physiological (single-unit or evoked-potential) responses to sounds with temporal interruptions.
}

important in temporal resolution and specifically in gap detection. Lesions of the auditory cortex have been shown to produce deficits in gap detection in rats and ferrets, animals whose temporal resolution is similar to that of humans (Ison, O'Conner, Bowen, \& Bocirnea, 1991; Kelly \& Rooney, 1996). Further, Shannon and Otto (1990) have reported that gap detection in people with auditory brainstem implants was about the same as, or perhaps a little worse than, that of people with normal hearing or with cochlear implants. That gap detection is unaffected when the periphery is compl etely bypassed suggests that the periphery may not be the limiting factor in normal processing, but at the very least that central mechanisms are also involved. Finally, a recent model of temporal processing that includes a bank of modulation filters following peripheral processing has done an excellent job of predicting psychophysical results with real istic cochlear filtering (Dau, Kollmeier, \& Kohlrausch, 1997a, 1997b).

We were interested in differentiating peripheral and central sources of infants' poor gap detection thresholds (Trehub, Schneider, \& Henderson, 1995; Werner et al., 1992) by comparing infants' gap detection thresholds to their neural gap thresholds. The auditory brainstem response (ABR) seemed particularly well suited to this task. Aside from the cessation of neural activity during a temporal gap in a sound, the onset response of auditory nerve fibers appears to be correl ated with gap duration (Fay, 1985; Klump \& Gleich, 1991). At the level of the inferior colliculus, neurons that respond phasically beginning at the onset of sound are more sensitive to temporal gaps than other types of neurons (Walton et al., 1997). The importance of these observations in the present context is that the ABR is presumed to reflect the synchronous phasic onset response of auditory nerve and brainstem neurons. Thus, it seems likely that the amplitude of the ABR recorded at the onset of sound following a gap would vary systematically with gap duration, allowing calculation of a neural gap threshold. In fact, evoked potentials have been used in that fashion to establish neural gap thresholds of gerbils (Boettcher, Mills, Swerdloff, \& Holley, 1996) and dolphins (Popov \& Supin, 1997).

The purpose of this study was to compare the characteristics of ABR gap threshold to those of gap detection threshold in humans with a view toward applying theABR tothe study of gap detection devel opment. First, we estimated gap detection thresholds and ABR gap threshold in a group of adults with normal hearing to determine whether the two types of threshold were related. Second, we manipulated stimulus conditions using vari ous types of maskers to determine whether such manipulations had similar effects on ABR gap threshol ds and gap detection threshol ds in adults with normal hearing. Finally, we estimated gap detection thresholds 
and ABR gap thresholds in infants and in a few participants with hearing loss to determine whether the effects of immaturity and hearing loss were similar for the two types of threshold. The results of these experiments would allow us to determine whether the ABR would be a useful tool in the study of gap detection in general, and to make preliminary observations on the contributions of peripheral and brainstem auditory system contributions to gap detection by mature and immature listeners.

\section{Experiment 1: ABR Gap Thresholds and Gap Detection Thresholds}

\section{Method \\ Participants}

$A B R$ and psychophysical thresholds were obtained from 35 participants who ranged in age from 18 to 30 years. The test ear of all participants passed screening tympanometry at each test session, passed screening audiometry at octave frequencies from .5 to $8 \mathrm{kHz}$ at 15 $\mathrm{dB} \mathrm{HL}$, and demonstrated a click-evoked ABR at $20 \mathrm{~dB}$ $\mathrm{nHL}$. All participants were paid for participating.

\section{Stimuli}

Broadband noise (low-pass filtered at $7 \mathrm{kHz}$ ) was presented through an insert transducer (Etymotic ER-1) at one of three spectrum levels $\left(\mathrm{N}_{0}\right), 10,30$ or 50 $\mathrm{dB} S \mathrm{SP} / \mathrm{Hz}$. Temporal gaps were created by gating the noise ( $100 \%$ attenuation) for a specified duration $(\Delta t)$ with a rise/fall time measured at the output of the transducer of about $0.5 \mathrm{~ms}$. Gap duration could range from 0 to $125 \mathrm{~ms}$, in increments of $1 \mathrm{~ms}$. All testing was completed in a single-walled sound-attenuating booth, and stimuli were presented monaurally.

\section{ABR Recording and Threshold Estimation}

Scalp potentials were recorded (midline foreheadto-ipsilateral mastoid) with disposable silver/silverchloride electrodes. The contralateral mastoid served as ground. Interelectrode impedance was maintained at $\leq 5$ $k \Omega$. Bioelectric activity was amplified $\left(2 \times 10^{5}\right)$ and filtered $(100-3000 \mathrm{~Hz})$. This activity was fed to a high-speed data acquisition board (Data Translation DT2821) controlled by locally developed software and averaged over 2048 stimulus presentations. Two 15-ms noise bursts separated by $\Delta$ t constituted one stimulus presentation; the interstimulus interval was $50 \mathrm{~ms}$. Signal averaging was synchronized to the leading edge of the noise burst following the temporal gap by a trigger pulse generated at noise onset. ${ }^{2}$ All responses were replicated. Recordings were made only when participants were sleeping or resting quietly; ongoing EEG activity was monitored on an oscilloscope. Artifact rejection was set to exclude from the averaged response any momentary voltage exceeding thefull scaleA/D range $( \pm 1 \mathrm{~V})$. Averageresponses were coded and stored on disk for offline analysis.

The person collecting the data decided whether or not a response was present after each recording. For this study, the ABR was defined by the presence or absence of its principal component, Wave $V$. A descending series of $\Delta t$ was presented until Wave $V$ was no longer apparent in the response. The first response was re corded for $\Delta \mathrm{t}$ of $30 \mathrm{~ms}$. $\Delta \mathrm{t}$ was decreased in steps of 10 $\mathrm{ms}$ to $10 \mathrm{~ms}$, then to $5 \mathrm{~ms}$, and finally to $1 \mathrm{~ms}$, as necessary. When Wave $V$ was judged to no longer be present, $\Delta$ t was increased in 1-ms steps until Wave $V$ was clearly re-established. Examples of ABRs recorded for a series of $\Delta \mathrm{t}$ are shown in Figure 1.

The final estimate of ABR gap threshold was assigned offline. Two experienced judges, blind to stimuI us condition, examined the aggregate of the replicated response waveform and decided whether or not Wave $V$ was present. A response was considered to be present only if the observers agreed in their judgments. A third experienced observer, also blind to stimulus conditions, arbitrated disagreements between judges. Gap threshold was taken as the midpoint between the lowest $\Delta t$ at which Wave $V$ was considered to be present and the highest $\Delta \mathrm{t}$ at which Wave $\mathrm{V}$ was considered to be absent.

\section{Psychophysical Method}

Because we planned to compare results of infants and adults, the adults in this preliminary study were tested in one of two different psychophysical methods. The first was the standard 2AFC paradigm; the other was the go/no-go procedure that we typically use to obtain infant thresholds. Whether these two methods produced similar gap detection thresholds and whether those gap detection thresholds were similarly related to ABR gap-detection threshold was a question of interest. I n both methods, however, stimuli were presented for a longer period of timethan they would have been in a typical psychophysical study, because longer durations

\footnotetext{
${ }^{2}$ Responses from offset $A B R s$, resulting from termination of the noise burst preceding the temporal gap, could potentially have contaminated the onset response. To rule out this possibility, recordings were made triggered at the offset of a single noise burst in pilot work. No offset responses were detectable when using high-pass filter settings of $100 \mathrm{~Hz}$ and maintaining stimulus levels of $50 \mathrm{~dB} \mathrm{SPL} / \mathrm{Hz}$ and below.
} 
Figure 1. Examples of ABR waveforms recorded from an adult (left panel) and from a 3-month-old infant (right panel) following temporal gaps of various durations.

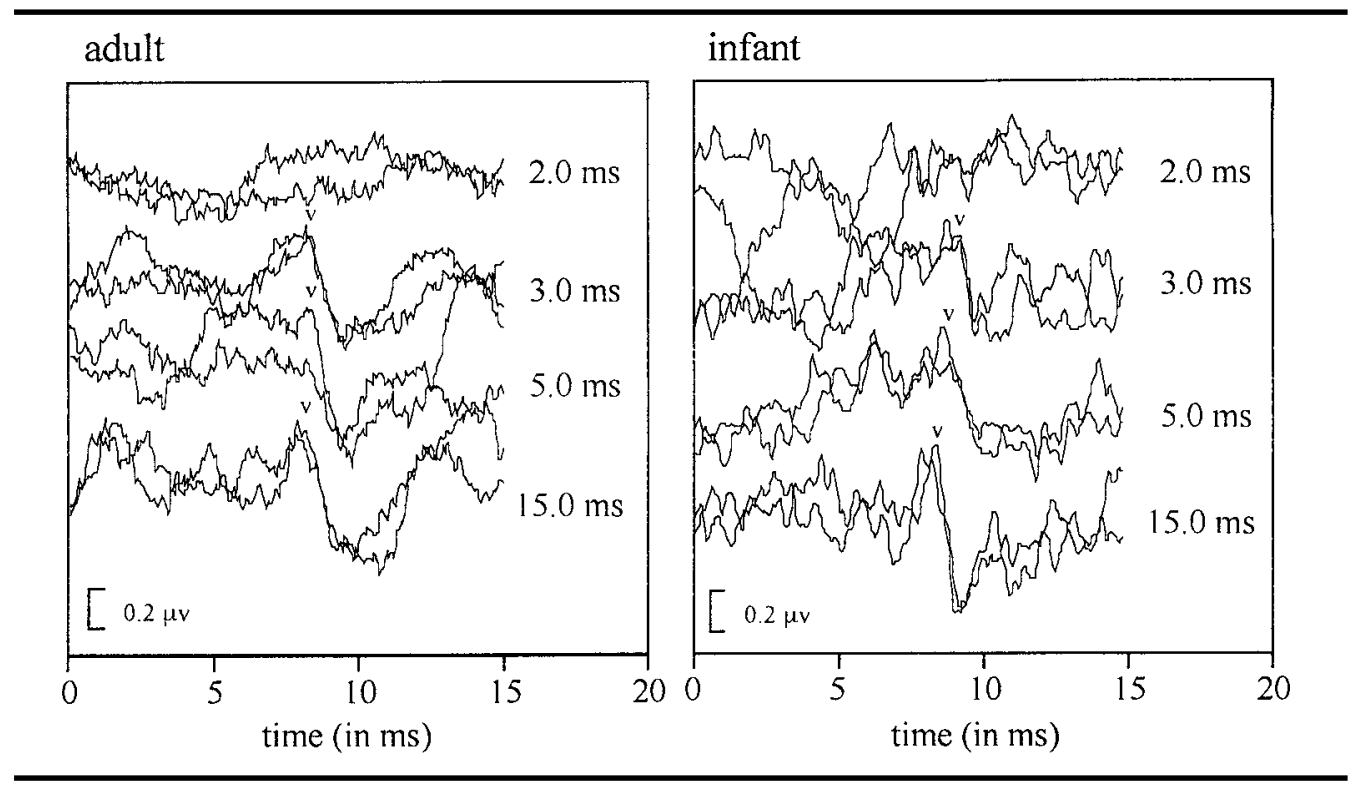

are required to give infants, particularly 3-month-olds, time to respond to signals. Long stimuli were used in both procedures to make the duration of stimulation similar.

\section{AFC}

Two 3,000-ms bursts of broadband noise at $30 \mathrm{~dB}$ $\mathrm{N}_{0}$ were presented, separated by an interval of $1000 \mathrm{~ms}$. A temporal gap was temporally centered in one of the two noi se bursts, randomly chosen. The partici pant was instructed to choose the interval that contained the gap. Gap duration was varied adaptively according to a oneup, two-down rule, beginning at $125 \mathrm{~ms}$. Step size was determined using PEST rules (Taylor \& Creelman, 1967). Testing was continued until 12 reversals were obtained, in about 50 trials. Gap detection threshold was calculated as the average of the last 10 reversals. Ten partici pants provided both $A B R$ and $2 A F C$ psychophysical gap detection thresholds.

\section{Go/No-Go Procedure}

When the participant appeared to be ready, an experimenter watching from outside the booth began a trial. On each trial, the broadband noise, at either 10, 30 , or $50 \mathrm{~dB} \mathrm{~N}_{0}$, was presented for a duration, in $\mathrm{ms}$, equal to $12(\Delta t+700)$. On signal trials, 12 gaps of duration $\Delta$ t occurred separated by intervals of $700 \mathrm{~ms}$. On no-signal trials, the noise was presented continuously. Signal and no-signal trials occurred with equal probability. The participant was instructed to respond to "the sound that makes the toy come on" by raising a hand. A mechanical toy in a dark Plexiglas box, used in the testing of infants, was illuminated for 2 seconds as feedback when the participant correctly responded on a signal trial. No feedback occurred following the trial if the participant failed to respond on a trial or if the participant responded on a no-signal trial. The gap duration was varied adaptively following the same procedure as in 2AFC testing. Gap detection threshold was taken as the last 8 of 10 reversals. On average, a threshold was based on 39.7 trials ( $S D=8.4$ trials). Each participant listened at only one level; 7 participants listened at $10 \mathrm{~dB} \mathrm{~N} \mathrm{~N}, 6$ participants listened at $30 \mathrm{~dB} \mathrm{~N}$, and 12 participants listened at $50 \mathrm{~dB} \mathrm{~N}$. Each participant'sABR gap threshold was determined at the signal level tested psychophysically.

\section{Results}

\section{Effects of Psychophysical Method}

The average gap detection threshold for the 10 adults tested with the $2 \mathrm{AFC}$ method at $30 \mathrm{~dB} \mathrm{~N}_{0}$ was $2.6 \mathrm{~ms}$ (SEM $=0.3$ ). The average gap detection threshold for the six adults tested with the go/no-go procedure at $30 \mathrm{~dB} \mathrm{~N}$ was $2.7 \mathrm{~ms}$ (SEM $=0.2$ ). This difference was not statistically significant (by t test, $p=.9$ ). Although one might have predicted that the go/no-go thresholds should have been lower than the 2AFC thresholds because more gaps were presented on each trial in the go/ no-go procedure, the effects of response bias or other procedural differences may have offset the increase in the number of "looks." The data of the two groups were, in any case, combined in subsequent analyses. 


\section{ABR Versus Psychophysical Average Threshold}

ABR gap thresholds and psychophysical gap detection thresholds were, on average, quitesimilar. Collapsed across levels, the average electrophysiological threshold was $2.4 \mathrm{~ms}$ (SEM $=0.1$ ), whereas the average psychophysical threshold, including both psychophysical methods, was $2.9 \mathrm{~ms}$ (SEM =0.2). The average thresholds estimated electrophysiologically and psychophysically are shown as a function of signal level in Figure 2 . The two types of threshold are similar, although a tendency for the ABR threshold to be lower than the psychophysical threshold is evident at each signal level. A 2 (Measure: Psychophysical, ABR) × 3 (Level: 10, 30, $50 \mathrm{~dB}$ ) analysis of variance with repeated measures on the Measure variable was performed to test the significance of the apparent differences. The Measure $\times$ Level interaction was not significant $(p=.15)$. The effects of Measure ( $p=.001)$ and of Level $(p=.028)$ were significant. Post hoc analyses indicated that thresholds at $10 \mathrm{~dB} \mathrm{~N}$ were significantly greater than those at higher levels, but that the 30 and $50 \mathrm{~dB} \mathrm{~N}$ thresholds did not differ. Thus, ABR gap threshold tends to be somewhat lower than psychophysical gap detection threshold, but ABR and psychophysical thresholds are similarly affected by signal level.

\section{Relationship Between ABR and Psychophysical Threshold}

Another question is whether an individual's psychophysical threshold can be predicted from that individual's ABR threshold. To answer that question, the data of all 35 participants were included in the correlational analyses. To exclude the possibility that level effects on gap

Figure 2. Mean gap detection threshold and ABR gap threshold as a function of spectrum level. Error bars represent \pm 1 standard error.

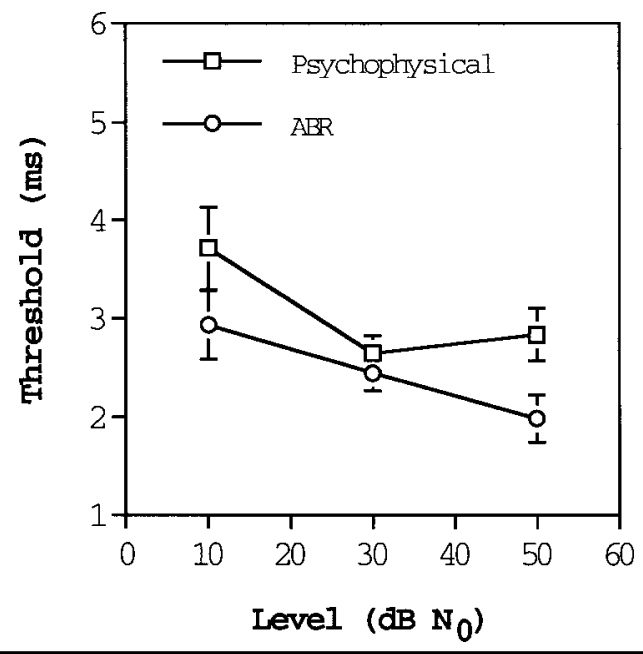

detection threshold mediate the correlation, the partial correlation between ABR and psychophysical threshold controlling for level was calculated. The partial correlation was 0.39 and statistically significant $(p=.011)$. Thus a modest relationship between ABR gap threshold and gap detection threshold exists in young, individuals with normal hearing. To illustrate that relationship, a scatterpl ot of residual scores for the two types of measure is shown in Figure 3. The residual scores plotted in this figure have had the effects of level "removed."

\section{$\overline{\text { Discussion }}$}

The average ABR gap threshold was similar to the average gap detection threshold obtained here and to gap detection thresholds for broadband signals reported in many other studies (Viemeister \& Plack, 1993). The increase observed in both psychophysical gap detection threshold and ABR gap threshold at low signal level has been reported in previous gap detection studies (e.g., Fitzgibbons, 1983).

Three results of Experiment 1 argue that psychophysical gap detection and the ABR gap threshold share a dependence on at least some processes: the similarity in the value of the threshol ds; their common dependence on signal level; and the significant, if modest, correlation between the two types of thresholds across individuals. These findings suggest that the temporal aspects of the peripheral or brainstem response, such as across-fiber discharge synchrony and adaptation, are reflected in gap detection. In addition, these findings suggest that

Figure 3. Partial correlation between gap detection threshold and A BR gap threshold controlling for spectrum level. "Residual" refers to the threshold that cannot be accounted for by spectrum level. Light gray points indicate two overlapping data points; dark gray points indicate three overlapping data points.

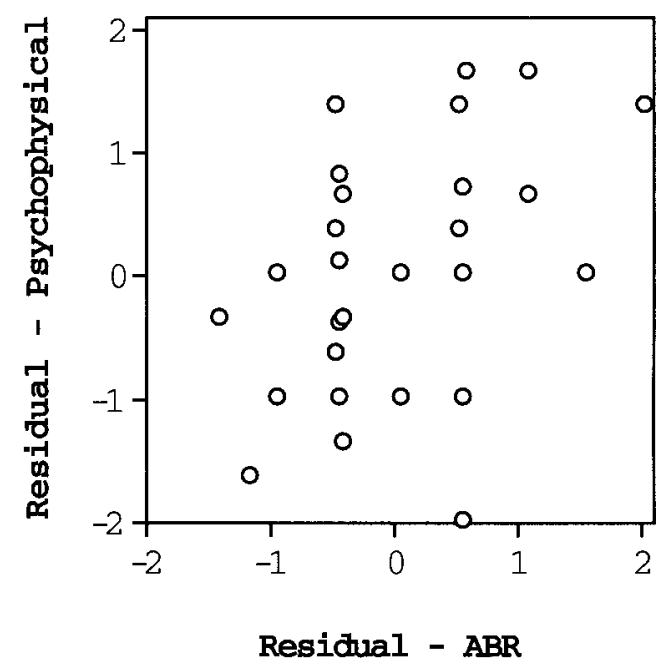


the ABR may bea useful tool in determining how peripheral or brainstem immaturities contribute to immature gap detection in human infants, although it would clearly be difficult to predict gap detection threshold from an individual's ABR gap threshold.

The reader may be puzzled by the finding that $A B R$ gap threshold is at least as good as the psychophysical gap detection threshold. After all, it is well established that thresholds for detecting sound are lower than the lowest levels of sound that reliably elicit an ABR. Keep in mind, though, that the ABR is highly dependent on the synchronous discharge of neurons at the onset of thestimulus in all stimulus configurations, whereas the neural "evidence" that listeners use to perform a psychophysical task will change with the task. Thus, listeners may be able to detect sounds when there is not enough synchronous neural acti vity to produce a recordable ABR. The similarity of ABR gap thresholds and psychophysical gap detection thresholds suggests that listeners may depend on neural synchrony, or a neural response characteristic that is highly correlated with synchrony, when they are detecting gaps. It is also possible that the peripheral and brainstem portions of the auditory nervous system represent the temporal characteristics of sound with greater fidel ity than do the levels of the system accessible to the decision-making process. Although single-unit studies generally report gap thresholds that are similar to gap detection thresholds, less forward masking, a phenomenon closely related to gap detection, has been observed in the peripheral neural response than was observed psychophysically when a signal detection analysis was employed to describe sensitivity (Relkin \& Turner, 1988). Finally, it would be difficult to argue that the $A B R$ and psychophysical thresholds reflect the same underlying process were their values vastly different, but there is no particular reason to expect the values to be exactly equal. Thresholds are by definition arbitrary. One could easily make the ABR gap threshold a half millisecond longer by defining the threshold as the midpoint between the lowest $\Delta \mathrm{t}$ at which Wave $\mathrm{V}$ was considered to be present and the highest $\Delta t$ at which Wave $V$ was considered to be absent, plus $0.5 \mathrm{~ms}$. Moreover, thefunction relating ABR amplitude to gap duration and the function relating gap detection to gap duration may bequite different in form, even if they reflect common processes.

Although the results of Experiment 1 suggest that the ABR gap threshold is measuring some of the same processes that determine the gap detection threshold, evoked potentials and psychophysical responses are obviously different and are affected by different "nuisance variables." Stimulus manipulations that one might want to make to examine the characteristics of temporal processing could make it difficult to record an ABR even though temporal processing has been unaffected by the manipulations. Varying stimulus frequency is one such manipulation. In psychophysical studies of gap detection, frequency is often manipulated by using continuous filtered noise to mask frequencies other than the ones that the experimenter wants to test. Maskers may be used to prevent listeners from using spectral splatter associated with rapid switching of frequency-specific sounds to detect gaps or to limit the range of informative frequencies in a broadband sound. It is not known how such maskers and the resulting frequency restriction would affect the ABR gap threshold.

\section{Experiment 2: Effects of Masking on ABR Gap Thresholds and Gap Detection Thresholds}

In Experiment 2, the effects of masking on ABR gap thresholds and gap detection threshol ds were examined in young adults with normal hearing. The effects of both frequency and bandwidth on the thresholds were of interest, because although temporal resolution has been found to depend on bandwidth, but not frequency, in adults (Eddins, 1993, 1999; Eddins, Hall, \& Grose, 1992), it is well known that thefrequency content of the stimulus strongly affects the ABR. First, ABR gap threshold and gap detection threshold were examined in the presence of high-pass noise. In this condition, both the fre quencies and the bandwidth available to generate a response varied with the cutoff frequency of the masker. Second, both types of thresholds were examined in the presence of notched-noisemaskers with notches centered at either 1.5 or $5 \mathrm{kHz}$. In this condition, the frequencies available to generate a response varied, but the available bandwidth (in $\mathrm{Hz}$ ) was held constant. The effects of level and signal-to-noise ratio werealso examined for the notched-noise maskers to identify conditions under which ABR gap thresholds could be reasonably obtained.

\section{$\overline{\text { Method }}$}

\section{Participants}

One hundred thirty adults with normal hearing, aged 18-30 years, participated. All participants met the same criteria for partici pation as in Experiment 1. Each participant provided both ABR and psychophysical data. The data of the participants who partici pated in Experiment 1 are al so considered in the analysis.

\section{Stimuli}

In all conditions, the interrupted stimulus (in which the participant detected gaps) was a noise. This noise is 
referred to as the signal. One group of 14 participants detected gaps in the signal in the presence of a continuous high-pass masker, with a low-frequency cutoff of either 1 or $2 \mathrm{kHz}$. The nominal filter slope was $96 \mathrm{~dB} /$ octave. Signal level was $30 \mathrm{~dB} \mathrm{~N}$, and masker level was $40 \mathrm{~dB} \mathrm{~N} \mathrm{~N}_{0}$. The signal noise was low-pass filtered at 7 $\mathrm{kHz}$, as in Experiment 1. A second group of 116 participants detected gaps in the presence of a continuous notched noise. The spectral notches were centered at either 1.5 or $5 \mathrm{kHz}$, and were $2 \mathrm{kHz}$ wide. The notched noises were produced by multiplying each of two lowpass (96 dB/octave) noises by a sinusoid to produce two broad bands of noise with nominal skirt slopes greater than $200 \mathrm{~dB}$ /octave (Patterson, 1974). The noise bands were $0.7 \mathrm{kHz}$ wide for the $1.5-\mathrm{kHz}$ notch and $1 \mathrm{kHz}$ wide for the $5-\mathrm{kHz}$ notch. In the $1.5-\mathrm{kHz}$ notch condition, the signal noise was bandpass filtered between 0.5 and 2.5 $\mathrm{kHz}$; in the 5-kHz notch condition, the signal noise was bandpass filtered between 4 and $6 \mathrm{kHz}$. Half of the participants were tested at a signal-to-masker ratio of 0 $\mathrm{dB}$, and signal level was 10,30 , or $50 \mathrm{~dB} \mathrm{~N}_{0}$, with 9-10 participants tested at each Frequency $\times$ Level combination. The rest of these participants were tested at a signal level of $30 \mathrm{~dB} \mathrm{~N}$, and signal-to-masker ratio was $-5,0$ or $5 \mathrm{~dB}$, again with 9-10 participants in each cell. The between-participants design was used to make it possible to examine correlations between measures without violating the assumption of independence among observations; that is, the same participant could not beincluded in a correlational analysis more than once.

\section{Procedure}

The ABR and psychophysical test procedures were the same as those used in Experiment 1, except that the
ABR filter cutoffs were 30 and $3000 \mathrm{~Hz}$ for the 1.5- and 5-kHz notched-noise masker conditions. Each participant listened in just one stimulus condition, but was tested both psychophysically and electrophysi ol ogically in that condition.

\section{Results \\ High-Pass Masker}

The effects of the high-pass maskers were assessed by comparing thresholds in the high-pass masked condition to the unmasked thresholds obtained at $30 \mathrm{~dB} \mathrm{~N}$. in Experiment 1. The average thresholds in all conditions are shown in Figure 4. Introducing a high-pass masker increased both ABR and psychophysical thresholds. A 2-kHz high-pass masker increased thethreshold from about 3 to about $10 \mathrm{~ms}$ and a $1-\mathrm{kHz}$ high-pass masker to about $20 \mathrm{~ms}$. There is a tendency for ABR threshold to be affected more than psychophysical threshold, but statistical analysis failed to givesubstance to that tendency. Nonparametric analyses were used because variability was higher in the masked conditions than in the unmasked condition. The effects of masking condition weresignificant for both ABR and psychophysical threshold, as indicated by Kruskal-Wallis one-way ANOVA ( $p<.00001$ in both cases). Friedman two-way ANOVA comparisons between measures in each masking condition indicated no difference between measures in either the 1-kHz or 2-kHz high-pass masker condition ( $p>.05$ in both cases). Although the difference between measures in the unmasked conditions was significant ( $p=.005$ by Friedman two-way ANOVA; see also results of Experiment 1), the difference was only a fraction of a millisecond. Thus, there is little statistical

Figure 4. Mean gap detection threshold and ABR gap threshold in unmasked and two masked conditions. Error bars represent \pm 1 standard error.

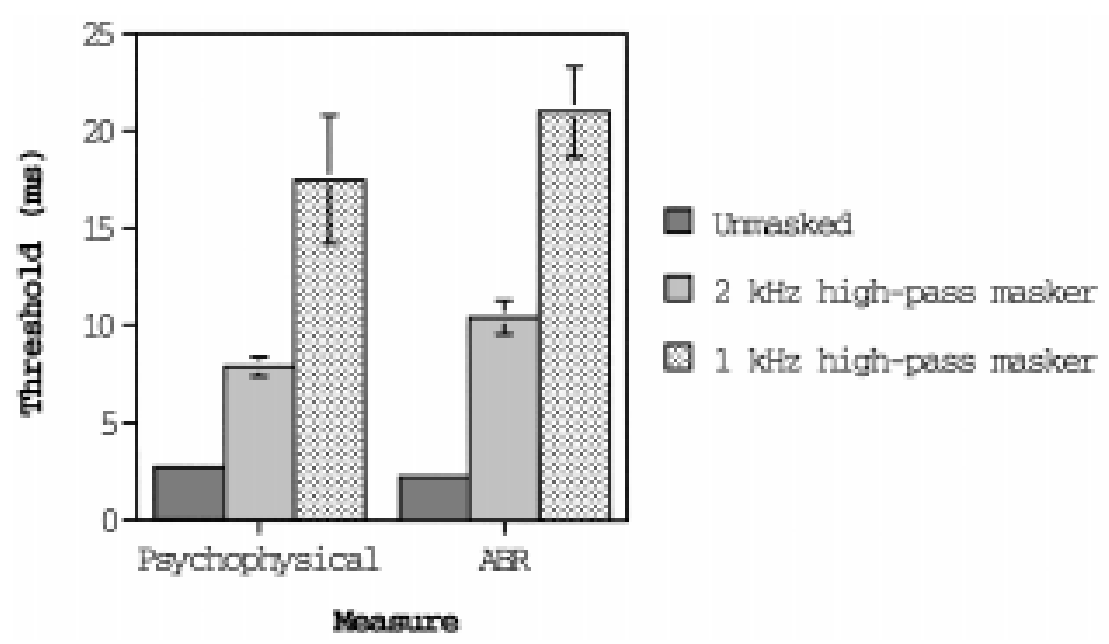


evidence here for a more pronounced effect of high-pass masking on the ABR measure compared to the psychophysical measure of gap detection.

The correlation between ABR and psychophysical threshold in the high-pass masker conditions, partialing out the effect of low-frequency cutoff, was moderate ( $r=$ $.6)$ and significant $(p=.03)$.

\section{Notched-N oise Masker: Effects of Frequency and Level}

The average ABR gap threshold and gap detection threshold in the presence of a notched-noise masker are shown as a function of spectrum level in Figure 5 . The most dramatic effect evident in these data is the interaction between the center frequency of the masker notch and spectrum level. The effects of level are pronounced for the ABR gap threshold measured in the presence of a $1.5-\mathrm{kHz}$ notched noise, whereas the effects of level are slight for the ABR threshold measured in the presence of a 5-kHz notched noise and for the psychophysical threshold in the presence of either notched noise. Except at the highest spectrum level, the 1.5-kHz notched-noise-masked ABR threshold is higher than that in any other condition. Thresholds in other conditions are in the 3- to 6-ms range.

Nonparametric statistical analyses were used because the variance differed across conditions, as is clear in Figure 5. First, the F requency $\times$ Level interaction was tested using the adjusted rank transform test suggested by Sawilowsky (1990). It was significant for the ABR ( $p$ $=.016$ ), but not for the psychophysical threshold ( $p=$ .67). Kruskal-Wallis one-way ANOVA showed that the effects of level on psychophysical threshold, although only on the order of 1-2 ms, were significant at both frequencies, with the $50 \mathrm{~dB} \mathrm{~N}_{0}$ threshold better than those at lower levels $(p=.03$ and .003 at 1.5 and $5 \mathrm{kHz}$, respectively). The level effect in Experiment 1 was similar in magnitude. The effects of level on ABR threshold were significant at $1.5 \mathrm{kHz}(p=.0008)$, but not at $5 \mathrm{kHz}$ $(p=.76)$. All pairwise differences between levels were significant at $1.5 \mathrm{kHz}$. The effects of frequency on the ABR threshold were significant $(p=.02)$, but the effects on the psychophysical threshold were not $(p=.73)$. The results of the statistical analysis are consistent with the above observations: The ABR gap threshold tends to be poor at $1.5 \mathrm{kHz}$, particularly at lower levels.

\section{Notched-N oise Masker: Effects of Frequency and Signal-to-Masker Ratio}

The average ABR gap threshold and gap detection thresholds are plotted as a function of signal-to-masker ratio for two notched noises for each measure in Figure $6 .^{3} \mathrm{~N}$ ot surprisingly, gap detection tends to be better at higher signal-to-masker ratio whether it is measured

\footnotetext{
${ }^{3}$ The same stimulus, $30 \mathrm{~dB}$ pressure spectrum level at $0 \mathrm{~dB}$ signal-tomasker ratio, was used to obtain the thresholds plotted for $30 \mathrm{~dB}$ pressure spectrum level in Figure 5 and for $0 \mathrm{~dB}$ signal-to-masker ratio in Figure 6 from two different groups of participants. The psychophysical thresholds for both notched-noise maskers and the ABR gap threshold for the 1.5-kHz notched-noise masker were similar for the two groups of participants. However, although the ABR gap threshold for the 5-kHz notched-noise masker in the level-manipulated group was about $2.5 \mathrm{~ms}$, the threshold in the signal-to-masker-ratio-manipulated group was nearly $11 \mathrm{~ms}$. We have no ready explanation for the difference in this condition, save sampling error. Two participants in the level-manipulated group had gap thresholds falling well above the range recorded in the signal-tomasker-manipulated group; elimination of the outliers reduced the average gap threshold in the signal-to-masker-manipulated group to about $5 \mathrm{~ms}$. The analysis of signal-to-masker ratio effects, however, produced the same results whether the outliers were included or not.
}

Figure 5. Mean gap detection threshold and ABR gap threshold as a function of spectrum level with notched-noise maskers centered at 1.5 and $5 \mathrm{kHz}$. Error bars represent \pm 1 standard error. In some conditions, the error bars are smaller than the plot symbol.

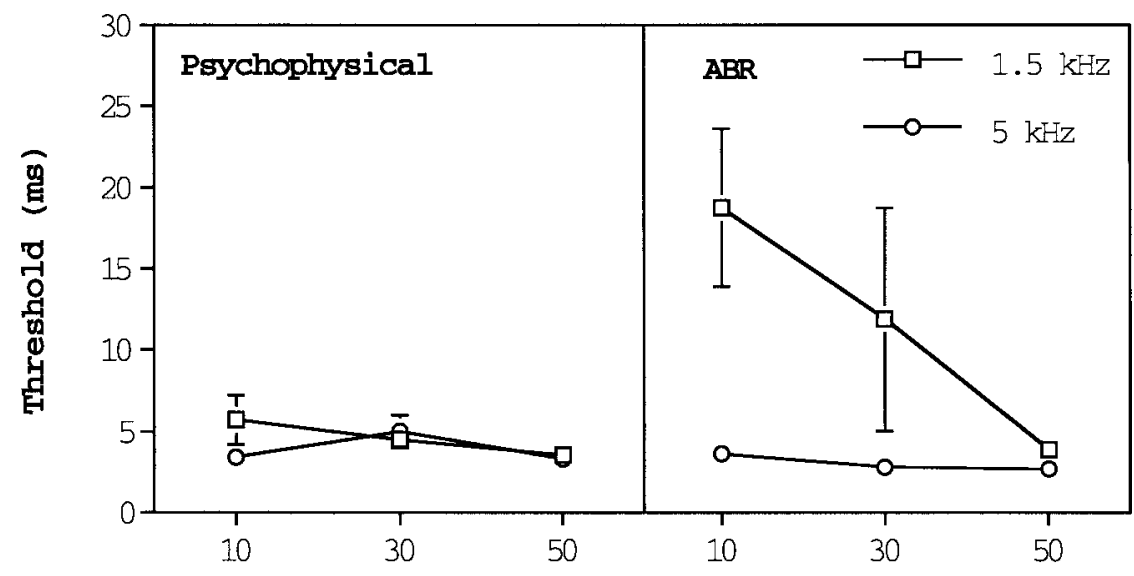

Level ( $\left.\mathrm{dB} \mathrm{N}_{0}\right)$ 
Figure 6. Mean gap detection threshold and $A B R$ gap thresholds as a function of signal-to-masker ratio with notched-noise maskers centered at 1.5 and $5 \mathrm{kHz}$.

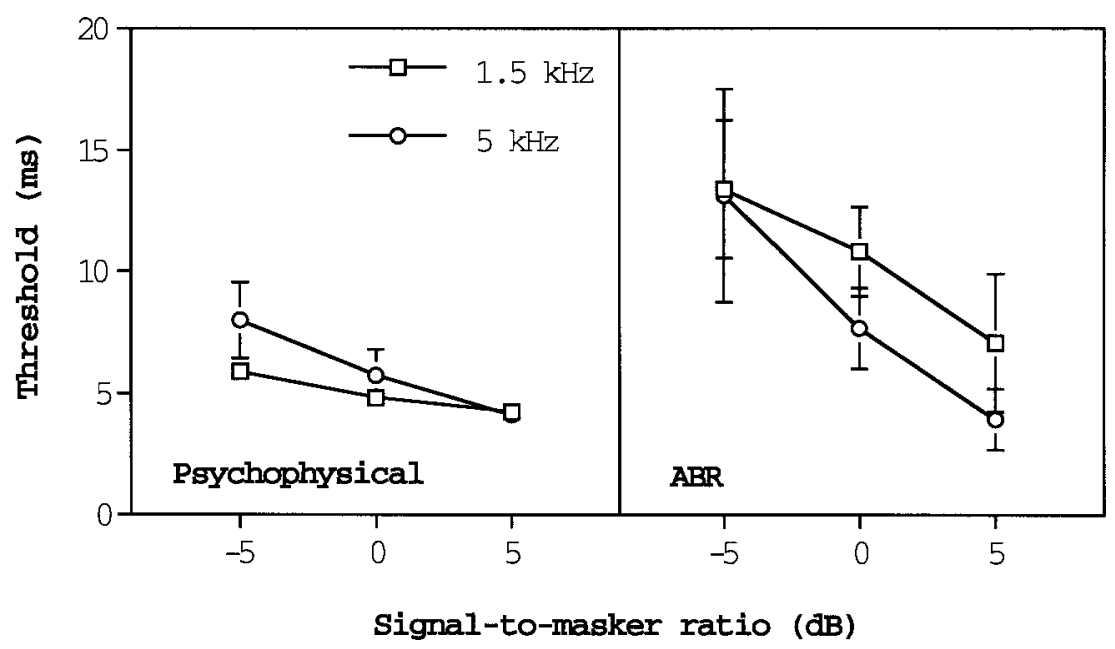

psychophysically or with $A B R$ in the presence of either a 1.5- or 5-kHz notched-noise masker. However, the effects of signal-to-masker ratio tend to be more pronounced for the ABR than for the psychophysical measure. Further, although the effects of signal-to-masker ratio on ABR gap threshold are similar for 1.5- and $5-\mathrm{kHz}$ notched-noise maskers, the effects on psychophysical gap threshold appear to be more pronounced for a $5-\mathrm{kHz}$ than for a $1.5-\mathrm{kHz}$ notched noise.

The significance of these effects was assessed using nonparametric statistics, again because the variance differed across conditions. The Frequency $\times$ Signal-toMasker Ratio effect was assessed for each measure using the adjusted rank transform test (Sawilowsky, 1990). Theinteraction was significant for neither the ABR $(p=$ .43) nor the psychophysical threshold $(p=.51)$. Thus, the apparent difference between frequencies in signalto-masker ratio effects on psychophysical gap detection was not significant. The effect of signal-to-masker ratio was significant for both ABR $(p=.004)$ and psychophysical threshold ( $p=.002$ ) by Kruskal-Wallis one-way ANOVA. However, the effect of Frequency was not significant for either measure (for both, $p>.05$ ).

To try to get at the relative importance of signal-tomasker ratio for the two measures, a robust linear regression was performed on threshold with signal-tomasker ratio the predictor variable for each measure. The slope of the line relating threshold to signal-tomasker ratio was 0.18 for the psychophysical threshold and 0.87 for the ABR threshold. The $95 \%$ confidence intervals were $0.08-0.28$ for the psychophysical threshold and 0.40-1.34 for the ABR threshold, consistent with the observation that signal-to-masker ratio has a greater effect on ABR gap threshold than on psychophysical measures of gap detection.
The correlation between ABR and psychophysical threshol d was examined for all the notched-noise masking conditions, partialing out the effects of notch center frequency, signal level, and signal-to-masker ratio. The correlation was not small and significant $(r=-.15, p=$ .17). The correlation between ABR gap threshold and gap detection threshold was also small and nonsignificant when only the data in more optimal $A B R$ conditions-5-kHz notch, $50 \mathrm{~dB} \mathrm{~N}$, or $+5 \mathrm{~dB}$ signal-to masker ratio-were considered.

\section{$\overline{\text { Discussion }}$}

A high-pass masker increases both the psychophysical and ABR gap threshol d from 2-3 ms in the unmasked case to 8-10 ms for a 2-kHz cutoff and 18-21 ms for a $1-\mathrm{kHz}$ cutoff. Along the same lines, a notched-noise masker increases either threshold to about $5 \mathrm{~ms}$ under the most favorable signal-to-masker ratio tested here. Gap thresholds in the presence of both high-pass noise and notched-noise maskers are similar to those reported for band-limited stimuli of the same bandwidth in previous psychophysical gap detection studies (reviewed by Eddins \& Green, 1995). As in those studies, the psychophysical gap detection thresholds in the presence of a notched-noise masker did not change with notch center frequency when bandwidth (in $\mathrm{Hz}$ ) was held constant. Further, the slight effect of level on psychophysical gap detection threshold is consistent with previous reports indicating little effect of level on gap at moderate or high sensation levels. The ABR gap thresholds in the presence of notched-noise maskers were also independent of notch center frequency when thestimuli were presented at a high enough level. 
At low signal levels, masker frequency affected gap detection and ABR gap threshold differently, however. The 1.5-kHz masker degraded ABR gap threshold more than it did gap detection, and more than did the $5-\mathrm{kHz}$ masker. The effects of bandwidth on gap detection have not been reported to interact with those of frequency in previous studies (E ddins \& Green, 1995). Considering that $1.5 \mathrm{kHz}$ is not an ideal stimulus for eliciting the $A B R$ to begin with, it is perhaps not surprising that the response to this frequency would be particularly degraded when the stimulus level is low.

One interesting outcome of Experiment 2 was that although the correlation observed between ABR gap threshold and gap detection threshold for unmasked broadband noi se was al so seen when high-pass maskers were used, it was not seen when notched-noi se maskers were used. The implication is that the notched-noise masker increased variability in the two measures differentially. Examination of $F$ igures 4 and 5 suggests that $A B R$ gap thresholds were generally more variable in notched-noise masker conditions compared to either masked gap detection threshold or to unmasked ABR gap threshold. Thus, it appears that the ABR to temporal interruptions may be particularly susceptible to disruption by notched-noise maskers. It is not entirely clear why the notched-noise maskers would affect the ABR more than high-pass maskers, especially when the stimulus bandwidth available to elicit the response is comparable in the two masking conditions. One possible explanation is that the upward spread of masking by the lower frequency band is particularly disruptive to theABR. However, it is not clear why the ABR would be more susceptible to such effects than psychophysical gap detection.

\section{Experiment 3: Effects of Subject Variables on ABR Gap Thresholds and Gap Detection}

Experiment 3 was intended to further explore the dependence of gap detection and ABR gap thresholds on common mechanisms. To the extent that the same factors limit the two measures, one would expect that partici pant variables such as age and hearing loss would affect the two measures in a similar way.

\section{Method}

\section{Participants}

\section{Participants With Hearing Loss}

Three adults with sloping, high-frequency, sensorineural hearing loss provided both the psychophysical and ABR gap thresholds. Ages for the participants with hearing loss ranged from 58 to 64 years. Their airconduction hearing thresholds are shown in Table1. The etiol ogy of hearing loss was presbycusis for $\mathrm{HI} 1$ and $\mathrm{HI}$, and presbycusis and noise exposure for $\mathrm{HI} 3$.

\section{Infants}

The infants tested psychophysically were ten 3month-old infants and twenty 6-month-old infants. Another thirteen 3-month-old infants were tested electrophysiologically. Infants were tested within 2 weeks of their 3-month or 6-month birthday. All participants passed a screening questionnair eassessing risk for hearing loss (J oint Committee on I nfant Hearing, 1991), had neither been treated for otitis media within 2 weeks nor had more than two episodes of otitis media, and passed screening tympanometry at each laboratory visit. None had participated previously in a psychoacoustic study. In the psychophysical part of the experiment, four additional 3-month-olds and three additional 6-month-olds completed the required test sessions, but either responded inconsistently to the probe or did not complete enough test trials to estimate a threshold.

I deally, we would test the sameinfants psychophysically and electrophysiologically. However, our experience in this arena has shown that considerable effort and expense are required to obtain sufficient data of that type, leading us to decideon a between-participants comparison for this initial study. Similarly, our decision not to obtain ABR thresholds from 6-month-ol ds was based on our initial observations of 3-month-olds'ABR thresholds and the fact that it is more difficult to get 6-montholds to fall into a natural sleep in the laboratory.

\section{Procedure}

The stimulus was the broadband noise used in Experiment 1 , unmasked and at a spectrum level of $30 \mathrm{~dB}$ $\mathrm{N}_{0}$. The procedures for recording the ABR and for estimating ABR gap thresholds were the sameas those used in Experiment 1, except that the infants were tested while in natural sleep. Infant electrophysiological test sessi ons typically lasted 1.5 hours, and thresholds were

Table 1. Air-conduction hearing thresholds ( $\mathrm{dB} \mathrm{HL}$ ) of 3 participants with hearing loss.

\begin{tabular}{crrrrrrrr}
\hline & \multicolumn{7}{c}{ Frequency (kHz) } \\
\cline { 2 - 9 } Participant & $\mathbf{0 . 2 5}$ & $\mathbf{0 . 5}$ & $\mathbf{1 . 0}$ & $\mathbf{2 . 0}$ & $\mathbf{3 . 0}$ & $\mathbf{4 . 0}$ & $\mathbf{6 . 0}$ & $\mathbf{8 . 0}$ \\
\hline HI1 & 10 & 10 & 5 & 10 & 30 & 45 & 65 & 65 \\
HI2 & 5 & 10 & 15 & 10 & 20 & 30 & 40 & 25 \\
HI3 & 0 & 0 & 15 & 45 & 45 & 55 & 50 & 50 \\
\hline
\end{tabular}


generally estimated in onesuch session. The psychophysical thresholds of listeners with hearing loss were estimated using the 2AFC procedure, as in Experiment 1.

The infants' psychophysical gap detection thresholds were estimated using the observer-based psychoacoustic procedure (Werner, 1995), which is a go/no-go procedure. The infant was seated on a parent's lap in the test booth, and an assistant in the booth maintained the infant in a mildly interested state by manipulating quiet toys on a table in front of the infant. As in Experiment 1, an experimenter observing from outside the booth began a trial when the infant appeared ready. On signal trials, a noise with gaps was presented; on nosignal trials, the continuous noise was presented. The observer knew when a trial was occurring, but not whether it was a signal trial. The observer decided, on the basis of observations of the infant's behavior, whether a signal or no-signal trial had occurred and received feedback on each judgment. If the observer correctly identified a signal trial, the mechanical toy was illuminated and activated in the booth to reinforce the infant's response to the noise with gaps. No reinforcement was presented following failures to respond or responses on no-signal trials. Thus, the infant learned to respond when the noise with gaps was presented, but not to the continuous noise. The observer learned to discriminate theresponse that theinfant made to the noise with gaps from the response that the infant made to the continuous noise.

At the beginning of each test session, easily detectable gaps were presented until the infant responded correctly $80 \%$ of the time on both signal and no-signal trials. Threshold was then estimated adaptively using the method described in the go/no-go condition of Experiment 1 . A threshold was only calculated if the infant completed 10 reversals. If the infant became too fussy or sleepy to continue testing, the training and threshold estimation procedure was repeated on a subsequent visit to the lab.

\section{Results \\ Effects of Hearing Loss}

As predicted, both psychophysical and electrophysiological thresholds were higher, on average, among the participants with hearing loss than among the participants with normal hearing. The psychophysical threshold averaged $10.7 \mathrm{~ms}$ (SEM = 5.7) for the participants with hearing loss compared to $2.6 \mathrm{~ms}$ for the participants with normal hearing. The ABR threshold averaged $12.7 \mathrm{~ms}$ (SEM =3.7) for the participants with hearing loss compared to $2.7 \mathrm{~ms}$ for the participants with normal hearing. Thus, the averagethresholds were similarly affected by sensorineural hearing loss.
There were differences among the 3 participants with hearing loss in the degree to which gap detection differed from normal, and the correlation between ABR gap threshold and gap detection threshold was not perfect. As shown in Figure 7, Participant HII had very poor gap detection thresholds, as estimated by both psychophysical and ABR testing, whereas Participant HI3's thresholds were less affected by hearing loss, although both thresholds were outside the normal range. However, Participant HI2 had a psychophysical threshold within the normal range, although the ABR threshold was well outside the normal range. However, the participant whose gap detection threshold was worst had the worst ABR gap threshold, and the participants whose gap detection was less affected by their hearing loss had better ABR gap thresholds.

\section{Effects of Age}

In contrast to the effects of hearing loss, the effects of early devel opment are very different for psychophysical and electrophysiological measures of gap detection. Although the average psychophysical gap detection threshold is an order of magnitude worse for 3- and 6month-olds than for adults, theaverageABR gap threshold is already adultlike by 3 months of age. The means are plotted in Figure 8. Nonparametric Kruskal-Wall is one-way analyses of variance were conducted to test the effect of age on each gap detection measure. A nonparametric statistic was chosen because of the differences in variance among age groups. For the psychophysical threshold, the effect of age was significant $(p<.0001)$; 3- and 6-month-olds had similar thresholds, but both

Figure 7. Mean gap detection threshold and ABR gap threshold for three participants with hearing loss. "Normal-hearing range" is the range of gap detection thresholds obtained for participants in Experiment 1 at $10 \mathrm{~dB} N_{0}$.

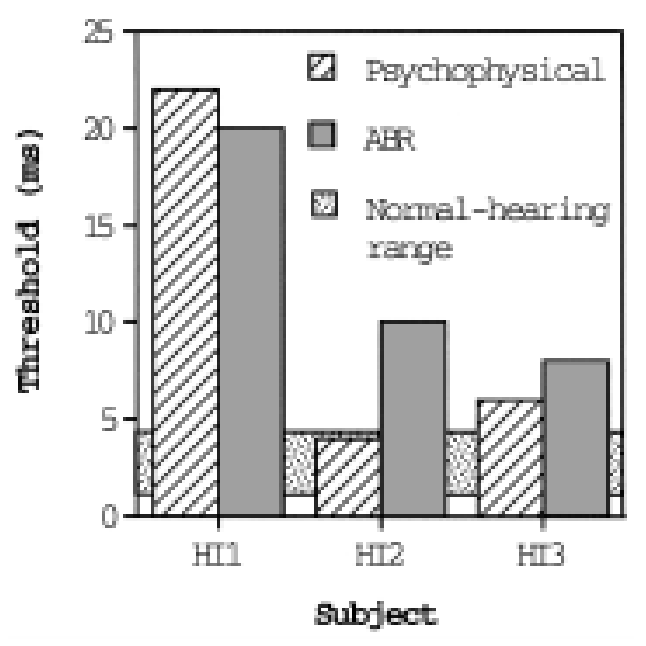


Figure 8. Mean gap detection threshold and $A B R$ gap thresholds at three ages. Error bars represent \pm 1 standard error. N o ABR gap thresholds were obtained for 6 -month-olds.

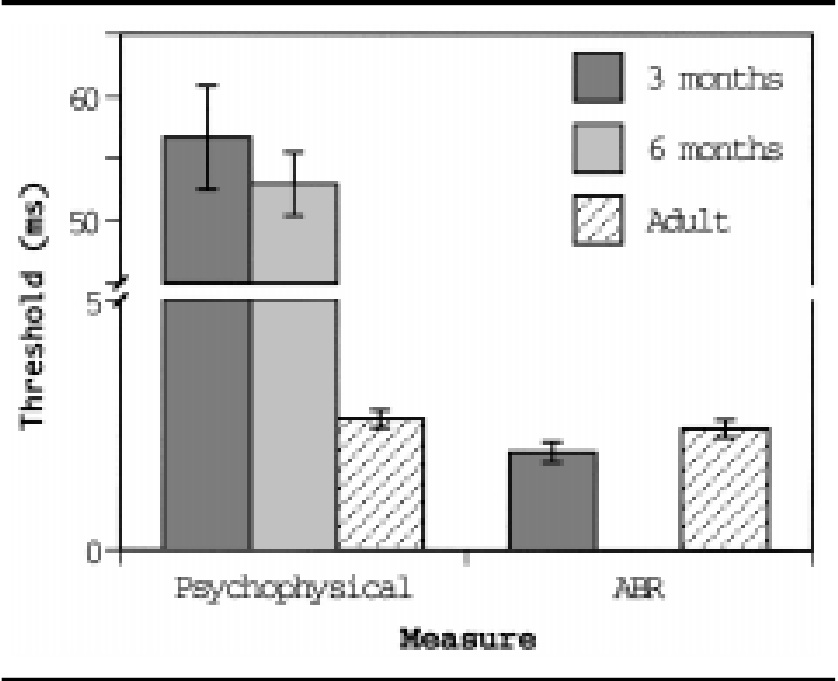

groups had significantly worse thresholds than did adults. For the ABR threshold, the effect of age was not significant $(p>.12)$.

\section{Discussion}

The psychophysical results of Experiment 3 are consistent with those of previous psychophysical studies. F or example, Nelson and Thomas (1997) and F itzgibbons and Gordon-Salant (1987) report similar values of gap detection thresholds for listeners with a sloping, highfrequency sensorineural hearing loss under similar stimulus conditions; and as in those studies, considerable variability is observed, even in this small group of listeners with hearing loss. Finally, the gap detection performance of infants in this study matches that previously reported by Werner et al. (1992) for gap detection in broadband noise.

The mechanisms involved in sensorineural hearing loss appear to affect gap detection threshold and electrophysiological gap threshold. The implication is that the peripheral factors responsiblefor sensorineural hearing loss constrain gap detection performance among listeners with hearing loss. Thus, the ABR may be a useful way to study temporal resolution in hearing loss. However, that one of the three listeners with hearing loss tested here could achieve normal gap detection threshold with abnormal ABR gap threshold is problematic. It suggests that gap detection can be based on neural information not reflected in theABR or that the ABR to gaps can be disrupted by mechanisms that do not affect psychophysical performance. Clearly, additional data would be needed to help to elucidate the mechanisms responsible for deficits in gap detection performance associated with sensorineural hearing loss.

The mechanisms involved in early auditory development appear to affect gap detection and el ectrophysiological gap threshold very differently. The implication is that the factors limiting psychophysical gap detection among infants are neither at the periphery nor in the brainstem. Given that implication, the ABR can contributelittle to an understanding of the immaturity that results in such poor gap detection among infants.

\section{General Discussion}

The first question asked in this study was the extent to which psychophysical gap detection depends on the peripheral and brainstem neural mechanisms that also contribute to the ABR. Several of the current results support the idea that at least some of the mechanisms that limit gap detection areat the periphery or in the brainstem. First, under most conditions, there is a significant correlation between ABR gap thresholds and gap detection thresholds, supporting a common dependence on some process. Moreover, ABR gap threshold and gap detection threshold are similar in value, are affected similarly by changes in signal level in the absence of masking, and by restrictions in the frequencies contributing totheresponse. F inally, sensorineural hearing loss appears to affect ABR gap detection as it does psychophysical gap detection.

I mmaturity does not affect ABR gap threshol ds and psychophysical gap detection thresholds in thesameway. Although the psychophysical gap detection threshold is clearly immature, the ABR gap threshold is not. The lack of correspondence between ABR gap threshol ds and psychophysical gap detection thresholds suggests that it is not immaturity at the level of the brainstem that is responsible for infants' poor gap detection performance. It should be noted that in previous work, we have found that immaturity of neural mechanisms that contribute little to frequency resolution or sensitivity in adults can result in immaturity of frequency resolution and sensitivity early in development (Folsom \& Wynne, 1987; Spetner \& Olsho, 1990; Werner, F ol som, \& Mand, 1993, 1994). Thus, although the auditory periphery may represent the temporal aspects of the stimulus in a mature fashion, that representation is either not maintained along the auditory pathway or not used efficiently by higher level processes. Along similar lines, Boettcher et al. (1996) reported changes in the gerbil ABR to gaps in the absence of changes in peripheral response.

Finally we ask whether the ABR is a useful tool in thestudy of temporal resolution. The general correspondence between $A B R$ and psychophysical results here 
indicates that it is, when the measure of temporal resolution is gap detection. At the same time, it is clear that one can manipulate the stimulus in such a way that the ABR to gaps is degraded, whereas psychophysical gap detection performance is little affected. Low signal-to-masker ratio and low levels in combination with a restriction to low frequencies are such manipulations. It appears that under these stimulus conditions, it be comes more difficult to record an ABR, probably for reasons that have more to do with the requirements of the $A B R$ than with auditory processing. However, given these limitations, the ABR gap threshold and psychophysical gap detection threshold remain similar when they are determined using sufficiently high levels and signal-to-masker ratio, even with low-frequency restriction. Thus, it appears that under most circumstances the ABR gives a reasonable prediction of psychophysical gap detection performance and should be useful in answering a variety of questions about the basis of gap detection.

\section{Author N ote}

This research was supported by NIDCD grants P01 DC00520 to R. C. Folsom and L. A. Werner and R01 DC00396 to L. A. Werner. J anelle Constantino collected some of the data described.

\section{References}

Boettcher, F. A., Mills, J . H., Swerdloff, J . L., \& Holley, B. L. (1996). Auditory evoked potentials in aged gerbils: Responses el icited by noises separated by a silent gap. Hearing Research, 102, 167-178.

Buchfellner, E., Leppelsack, H.-J ., Klump, G. M., \& Häusler, U. (1989). Gap detection in the starling (Sturnus vulgaris). II. Coding of gaps by forebrain neurons. J ournal of Comparative Physiology [A], 164, 539-549.

Dau, T., Kollmeier, D., \& KohIrausch, A. (1997a). Modeling auditory processing of amplitude modulation. I. Detection and masking with narrowband carriers. J ournal of the Acoustical Society of America, 102, 2892-2905.

Dau, T., Kollmeier, D., \& KohIrausch, A. (1997b). Modeling auditory processing of amplitude modulation. II. Spectral and temporal integration. J ournal of the Acoustical Society of America, 102, 2906-2919.

Dreschler, W. A., \& Leeuw, A. R. (1990). Speech reception in reverberation related to temporal resolution. J ournal of Speech and Hearing Research, 33, 181-187.

Dreschler, W. A., \& Plomp, R. (1980). Relation between psychophysical data and speech perception for hearingimpaired listeners. J ournal of the Acoustical Society of America, 68, 1608-1615.

Eddins, D. A. (1993). Amplitude modulation detection in narrow-band noise: Effects of absolute bandwidth and frequency region. J ournal of the Acoustical Society of America, 93, 470-479.
Eddins, D. A. (1999). Amplitude-modulation detection at low- and high-audio frequencies. J ournal of the Acoustical Society of America, 105, 829-837.

E ddins, D. A., \& Green, D. M. (1995). Temporal integration and temporal resolution. In B. C. J . Moore (Ed.), Hearing (pp. 207-242). San Diego, CA: Academic Press.

Eddins, D. A., Hall, J . W., \& Grose, J . H. (1992). The detection of temporal gaps as a function of frequency region and absolute noise bandwidth. J ournal of the Acoustical Society of America, 91, 1069-1077.

Eggermont, J . J . (1995). Neural correlates of gap detection and auditory fusion in cat auditory cortex. Neuroreport, 6 , 1645-1648.

E ggermont, J . J . (1999). Neural correlates of gap detection in three auditory cortical fields in the cat. J ournal of Neurophysiology, 81, 2570-2581.

Fay, R. R. (1985). Sound intensity processing by the goldfish. J ournal of the Acoustical Society of America, 78, 1296-1309.

Feng, A. S., Lin, W.-Y., \& Sun, L. (1994). Detection of gaps in sinusoids by frog auditory nerve fibers: I mportance in AM coding. J ournal of Comparative Physiology [A], 175, 531-546.

Fitzgibbons, P. J . (1983). Temporal gap detection in noise as a function of frequency, bandwidth, and level. J ournal of the Acoustical Society of America, 74, 67-72.

Fitzgibbons, P. J ., \& Gordon-Salant, S. (1987). Temporal gap resolution in listeners with high-frequency sensorineural hearing loss. J ournal of the Acoustical Society of America, 81, 133-137.

Folsom, R. C., \& Wynne, M. K. (1987). Auditory brain stem responses from human adults and infants: Wave $V$ tuning curves. J ournal of the Acoustical Society of America, 81, 412-417.

Forrest, T. G., \& Green, D. M. (1987). Detection of partially filled gaps in noise and the temporal modulation transfer function. J ournal of the Acoustical Society of America, 82, 1933-1943.

Green, D. M. (1973). Temporal acuity as a function of frequency. J ournal of the Acoustical Society of America, 54, 373-379.

Ison, J . R., O'Conner, K., Bowen, G. P., \& Bocirnea, A. (1991). Temporal resolution of gaps in noise by the rat is lost with functional decortication. Behavioral Neuroscience, 105, 33-40.

J oint Committee on I nfant Hearing. (1991). 1990 Position statement. Asha, 33(Suppl. 5), 3-5.

Kelly, J . B ., \& Rooney, B. J . (1996). Effects of bilateral auditory cortical lesions on gap-detection thresholds in the ferret (Mustela putorius). Behavioral Neuroscience, 110, 542-550.

Klump, G. M., \& Gleich, O. (1991). Gap detection in the E uropean starling (Sturnus vulgaris). III. Processing in the peripheral auditory system. J ournal of Comparative Physiology [A], 168, 469-476.

Moore, B. C. J . (1993). Temporal analysis in normal and impaired hearing. Annals of the New York Academy of Science, 682, 119-136.

Moore, B. C. J ., Glasberg, B. R., Plack, C. J ., \& Biswas, 
A. K. (1988). The shape of the ear's temporal window. J ournal of the Acoustical Society of America, 83, 1102-1116.

Nelson, P. B., \& Thomas, S. D. (1997). Gap detection as a function of stimulus loudness for listeners with and without hearing loss. J ournal of Speech, Language, and Hearing Research, 40, 1387-1394.

Patterson, R. D. (1974). Auditory filter shapes. J ournal of the Acoustical Society of America, 55, 802-809.

Phillips, D. P. (1999). Auditory gap detection, perceptual channels, and temporal resolution in speech perception. J ournal of the American Academy of Audiology, 10, 343-354.

Popov, V. V., \& Supin, A. (1997). Detection of temporal gaps in noise in dolphins: Evoked-potential study. J ournal of the Acoustical Society of America, 102, 1169-1176.

Relkin, E. M., \& Turner, C. W. (1988). A reexamination of forward masking in the auditory nerve. J ournal of the Acoustical Society of America, 84, 584-591.

Sawilowsky, S. S. (1990). N onparametric tests of interaction in experimental design. Review of Educational Research, 60, 91-126.

Shannon, R. V., \& Otto, S. R. (1990). Psychophysical measures from electrical stimulation of the human cochlear nucleus. Hearing Research, 47, 159-168.

Spetner, N. B., \& Olsho, L. W. (1990). Auditory frequency resolution in human infancy. Child Development, 61 , 632-652.

Taylor, M. M., \& Creelman, C. D. (1967). PEST: Efficient estimates on probability functions. J ournal of the Acoustical Society of America, 41, 782-787.

Trehub, S. E ., Schneider, B. A., \& Henderson, J . (1995). Gap detection in infants, children, and adults. J ournal of the Acoustical Society of America, 98, 2532-2541.

Viemeister, N. F. (1979). Temporal modulation transfer functions based upon modulation thresholds. J ournal of the Acoustical Society of America, 66, 1364-1380.
Viemeister, N. F., \& Plack, C. J . (1993). Time analysis. In W. A. Yost, A. N. Popper, \& R. R. Fay (E ds.), Human psychophysics (pp. 116-154). New York: Springer-Verlag.

Walton, J . P., Frisina, R. D., Ison, J . R., \& O'NeilI, W. E. (1997). Neural correlates of behavioral gap detection in the inferior colliculus of the young CBA mouse. J ournal of Comparative Physiology [A], 181, 161-176.

Werner, L. A. (1995). Observer-based approaches to human infant psychoacoustics. In G. M. Klump, R. J . Dooling, R. R. Fay, \& W. C. Stebbins (Eds.), Methods in comparative psychoacoustics (pp. 135-146). Boston: Birkhauser Verlag.

Werner, L. A., Folsom, R. C., \& Mancl, L. R. (1993). The relationship between auditory brainstem response and behavioral thresholds in normal hearing infants and adults. Hearing Research, 68, 131-141.

Werner, L. A., Folsom, R. C., \& Mancl, L. R. (1994). The relationship between auditory brainstem response latencies and behavioral thresholds in normal hearing infants and adults. Hearing Research, 77, 88-98.

Werner, L. A., Marean, G. C., Halpin, C. F., Spetner, N. B., \& Gillenwater, J . M. (1992). I nfant auditory temporal acuity: Gap detection. Child Devel opment, 63, 260-272.

Zhang, W., Salvi, R. J ., \& Saunders, S. S. (1990). Neural correlates of gap detection in auditory nerve fibers of the chinchilla. Hearing Research, 46, 181-200.

Received April 26, 1999

Accepted April 11, 2001

DOI : 10.1044/1092-4388(2001/058)

Contact author: L. A. Werner, PhD, University of Washington, Department of Speech and Hearing Sciences, 1417 N.E. 42nd Street, Seattle, WA 98105-6246. E-mail: lawerner@u.washington.edu 